\title{
On defining soft spaces by weak soft neighborhood systems
}

\author{
Taha Yasin Ozturk \\ Department of Mathematics, Faculty of Science and Letters, Kafkas University, Kars, Turkey \\ Received: 17 February 2016, Revised: 22 March 2016, Accepted: 11 April 2016 \\ Published online: 18 April 2016.
}

\begin{abstract}
In the present paper, we define the concepts of weak soft neighborhood space, soft $w^{s}\left(\widetilde{\varphi}, \widetilde{\varphi}^{\prime}\right)-$ continuous, soft $w^{s}$-continuous and soft $w^{s^{*}}$ - continuous on weak soft neighborhood spaces. Finally, we introduce their basic properties and some examples.
\end{abstract}

Keywords: Weak soft neighborhood systems, weak soft neighborhood spaces, soft $w_{\widetilde{\varphi}}^{s}-$ open sets, soft $w^{s}\left(\widetilde{\varphi}, \widetilde{\varphi}^{\prime}\right)-$ continuous, soft $w^{s}-$ continuous, soft $w^{s^{*}}-$ continuous.

\section{Introduction}

Molodtsov [9] defined the notions of soft set theory as a new mathematical tool for dealing with uncertainties in economics, engineering, social science, medical science, etc. Subsequently, many authors have introduced different operations and on soft sets and studied a lot of applications. Many authors have developed algebra areas of soft set theory, after $\mathrm{Akta}^{\mathrm{O}}$ and Çağman [1] introduced soft groups. In 2011, Shabir and Naz [11] defined soft topological spaces and introduced basic properties. There have been many studies in the field of soft topology by some authors [?],[2], [3], [5], [6], [10], [11], [12], [13], [14].

In [4], Császár defined the concept of generalized neighborhood systems and generalized topological spaces. Thomas and John introduced soft generalized topology, soft generalized neighborhood systems and some properties in [15]. In 2008, Min defined the weak neighborhood systems and introduced some properties. The weak neighborhood systems are generalized systems of open neighborhood systems but stronger than generalized neighborhood systems.

In this paper, we introduce the weak soft neighborhood systems on a soft set defined by using the concept of weak soft neighborhoods. Let us note that weak soft neighborhood space is independent of a soft neighborhood space. We prove that soft $w_{\widetilde{\varphi}}^{s}$-open sets induced by the weak soft neighborhood systems form a soft topology. Furthermore, we define the concepts of some soft continuity and connected soft interior and soft closure operators on weak soft neighborhood spaces.

\section{Preliminaries}

In this section we will introduce necessary definitions and theorems for soft sets. Molodtsov [9] defined the soft set in the following way. 
Let $X$ be an initial universe set and $E$ be a set of parameters. Let $P(X)$ denotes the power set of $X$ and $A, B \subseteq E$.

Definition 1. [9] A pair $(F, A)$ is called a soft set over $X$, where $F$ is a mapping given by $F: A \rightarrow P(X)$.

In other words, the soft set is a parameterized family of subsets of the set X. For $e \in A, F(e)$ may be considered as the set of e-elements of the soft set $(F, A)$, or as the set of e-approximate elements of the soft set, i.e.,

$$
(F, A)=\{(e, F(e)): e \in A \subseteq E, F: A \rightarrow P(X)\}
$$

Definition 2. [7] For two soft sets $(F, A)$ and $(G, B)$ over $X,(F, A)$ is called soft subset of $(G, B)$ if

(i) $A \subset B$ and

(ii) $\forall e \in A, F(e)$ and $G(e)$ are identical approximations.

This relationship is denoted by $(F, A) \widetilde{\subseteq}(G, B)$. Similarly, $(F, A)$ is called a soft superset of $(G, B)$ if $(G, B)$ is a soft subset of $(F, A)$. This relationship is denoted by $(F, A) \supseteq(G, B)$. Two soft sets $(F, A)$ and $(G, B)$ over $X$ are called soft equal if $(F, A)$ is a soft subset of $(G, B)$ and $(G, B)$ is a soft subset of $(F, A)$.

Definition 3. [7] The intersection of two soft sets $(F, A)$ and $(G, B)$ over $X$ is the soft set $(H, C)$, where $C=A \cap B$ and $\forall e \in C, H(e)=F(e) \cap G(e)$. This is denoted by $(F, A) \widetilde{\cap}(G, B)=(H, C)$.

Definition 4. [7] The union of two soft sets $(F, A)$ and $(G, B)$ over $X$ is the soft set, where $C=A \cup B$ and $\forall e \in C$,

$$
H(\varepsilon)= \begin{cases}F(e), & \text { if } e \in A-B \\ G(e), & \text { if } e \in B-A \\ F(e) \cup G(e) & \text { if } e \in A \cap B\end{cases}
$$

This relationship is denoted by $(F, A) \tilde{\cup}(G, B)=(H, C)$.

Definition 5. [7] A soft set $(F, A)$ over $X$ is said to be a NULL soft set denoted by $\Phi$ if for all e $\in A, F(e)=\emptyset$ (null set).

Definition 6. [7] A soft set $(F, A)$ over $X$ is said to be an absolute soft set denoted by $\tilde{X}$ iffor all e $\in A, F(e)=X$.

Definition 7. [11] The difference $(H, E)$ of two soft sets $(F, E)$ and $(G, E)$ over $X$, denoted by $(F, E) \backslash(G, E)$, is defined as $H(e)=F(e) \backslash G(e)$ for all $e \in E$.

Definition 8. [11] Let $Y$ be a non-empty subset of $X$, then $\tilde{Y}$ denotes the soft set $(Y, E)$ over $X$ for which $Y(e)=Y$, for all $e \in E$.

In particular, $(X, E)$ will be denoted by $\tilde{X}$.

Definition 9. [11] The complement of a soft set $(F, E)$, denoted by $(F, E)^{c}$, is defined $(F, E)^{c}=\left(F^{c}, E\right)$, where $F^{c}: E \rightarrow$ $P(X)$ is a mapping given by $F^{c}(e)=X \backslash F(e), \forall e \in E$ and $F^{c}$ is called the soft complement function of $F$.

Definition 10. [11] Let $\tau$ be the collection of soft set over $X$, then $\tau$ is said to be a soft topology on $X$ if

(i) $\Phi, \tilde{X}$ belongs to $\tau$;

(ii) the union of any number of soft sets in $\tau$ belongs to $\tau$;

(iii) the intersection of any two soft sets in $\tau$ belongs to $\tau$.

The triplet $(X, \tau, E)$ is called a soft topological space over $X$. 
Definition 11. [11] Let $(X, \tau, E)$ be a soft topological space over X. Then members of $\tau$ are said to be soft open sets in X.

Definition 12. [11] Let $(X, \tau, E)$ be a soft topological space over $X$. A soft set $(F, E)$ over $X$ is said to be a soft closed in $X$, if its complement $(F, E)^{c}$ belongs to $\tau$.

Proposition 1. [11] Let $(X, \tau, E)$ be a soft topological space over $X$. Then the collection $\tau_{e}=\{F(e):(F, E) \in \tau\}$ for each $e \in E$, defines a topology on $X$.

Definition 13. [11] Let $(X, \tau, E)$ be a soft topological space over $X$ and $(F, E)$ be a soft set over $X$. Then the soft closure of $(F, E)$, denoted by $\operatorname{cl}(F, E)$ is the intersection of all soft closed super sets of $(F, E)$. Clearly cl $(F, E)$ is the smallest soft closed set over $X$ which contains $(F, E)$.

Definition 14. [?] Let $(F, E)$ be a soft set over $X$. The soft set $(F, E)$ is called a soft point, denoted by $\left(x_{e}, E\right)$, if for the element $e \in E, F(e)=\{x\}$ and $F\left(e^{c}\right)=\varnothing$ for all $e^{c} \in E-\{e\}$ (briefly denoted by $\left.x_{e}\right)$.

Definition 15. [?] Two soft points $\left(x_{e}, E\right)$ and $\left(y_{e^{\prime}}, E\right)$ over a common universe $X$, we say that the points are different points if $x \neq y$ or $e \neq e^{\prime}$.

Definition 16. [?] The soft point $x_{e}$ is said to be belonging to the soft $\operatorname{set}(F, E)$, denoted by $x_{e} \widetilde{\in}(F, E)$, if $x_{e}(e) \in F(e)$, i.e., $\{x\} \subseteq F(e)$.

Definition 17. [?] Let $(X, \tau, E)$ be a soft topological space over $X$. A soft set $(F, E)$ in $(X, \tau, E)$ is called a soft neighborhood of the soft point $\left(x_{e}, E\right) \widetilde{\in}(F, E)$ if there exists a soft open set $(G, E)$ such that $\left(x_{e}, E\right) \widetilde{\in}(G, E) \widetilde{C}(F, E)$.

Definition 18. [6] Let $(X, \tau, E)$ and $\left(Y, \tau^{\prime}, E\right)$ be two soft topological spaces, $f:(X, \tau, E) \rightarrow\left(Y, \tau^{\prime}, E\right)$ be a mapping. For each soft neighbourhood $(H, E)$ of $\left(f(x)_{e}, E\right)$, if there exists a soft neighbourhood $(F, E)$ of $\left(x_{e}, E\right)$ such that $f((F, E)) \widetilde{\subset}(H, E)$, then $f$ is said to be soft continuous mapping at $\left(x_{e}, E\right)$.

If $f$ is soft continuous mapping for all $\left(x_{e}, E\right)$, then $f$ is called soft continuous mapping.

Definition 19. [6] Let $(X, \tau, E)$ and $\left(Y, \tau^{\prime}, E\right)$ be two soft topological spaces, $f: X \rightarrow Y$ be a mapping. If $f$ is a bijection, soft continuous and $f^{-1}$ is a soft continuous mapping, then $f$ is said to be soft homeomorphism from $X$ to $Y$. When a homeomorphism $f$ exists between $X$ and $Y$, we say that $X$ is soft homeomorphic to $Y$.

Definition 20. [3] Let $\left\{\left(\varphi_{s}, \psi_{s}\right):(X, \tau, E) \rightarrow\left(Y_{s}, \tau_{s}, E_{S}\right)\right\}_{s \in S}$ be a family of soft mappings and $\left\{\left(Y_{s}, \tau_{s}, E_{s}\right)\right\}_{s \in S}$ is a family of soft topological spaces. Then, the topology $\tau$ generated from the subbase $\delta=\left\{\left(\varphi_{s}, \psi_{s}\right)_{s \in S}^{-1}(F, E):(F, E) \in \tau_{s}, s \in S\right\}$ is called the soft topology (or initial soft topology) induced by the family of soft mappings $\left\{\left(\varphi_{s}, \psi_{s}\right)\right\}_{s \in S}$.

Definition 21. [4] Let $X$ be a nonempty set and $g$ be a collection of subsets of X. Then $g$ is called a generalized topology on $X$ if and only if

(i) $\emptyset \in g$,

(ii) $G_{i} \in g$ for $i \in I \neq \emptyset$ implies $\cup_{i \in I} G_{i} \in g$.

Definition 22. [4] Let $X$ be a nonempty set and $\psi: X \rightarrow P(P(X))$ satisfy $x \in V$ for $V \in \psi(x)$. Then $V \in \psi(x)$ is called a generalized neighborhood of $x \in X$ and $\psi$ is called a generalized neighborhood system on $X$.

Definition 23. [15] Let $\mu$ be the collection of soft set over $X$. Then $\mu$ is said to be a soft generalized topology on $X$ if

(i) $\Phi$ belongs to $\tau$;

(ii) the union of any number of soft sets in $\mu$ belongs to $\mu$.

The triplet $(X, \mu, E)$ is called a soft generalized topological space over $X$. 
Definition 24. [8] Let $X$ be a nonempty set and $\psi: X \rightarrow P(P(X))$. Then $\psi$ is called a weak neighborhood system on $X$ if it satisfies the following:

(i) For $x \in X$ and $V \in \psi(x), x \in V$,

(ii) $\operatorname{For} U, V \in \psi(x), V \cap U \in \psi(x)$,

(iii) For $x \in X, \psi(x) \neq \emptyset$.

Then the pair $(X, \psi)$ is called a weak neighborhood space on $X . V \in \psi(x)$ is called a weak neighborhood of $x \in X$.

\section{Weak soft neighborhood systems and its spaces}

Definition 25. Let $\widetilde{\varphi}: S S(X)_{E} \rightarrow P\left(S S(X)_{E}\right)$ be a mapping. Then $\widetilde{\varphi}$ is called a weak soft neighborhood system on $(X, E)$ if it satisfies the following conditions:

(i) For $x_{e} \widetilde{\in} S S(X)_{E}$ and $(V, E) \in \widetilde{\varphi}\left(x_{e}\right), x_{e} \widetilde{\in}(V, E)$;

(ii) $\operatorname{For}(U, E),(V, E) \in \widetilde{\varphi}\left(x_{e}\right),(U, E) \widetilde{\cap}(V, E) \in \widetilde{\varphi}\left(x_{e}\right)$;

(iii) For $x_{e} \tilde{\in} S S(X)_{E}, \widetilde{\varphi}\left(x_{e}\right) \neq \Phi$.

Then the triple $(X, \widetilde{\varphi}, E)$ is called a weak soft neighborhood space (briefly WSNS) on $(X, E)$. The soft set $(V, E) \in \widetilde{\varphi}\left(x_{e}\right)$ is called a weak soft neighborhood of $x_{e} \widetilde{\in} S S(X)_{E}$.

Definition 26. Let $(X, \widetilde{\varphi}, E)$ be a WSNS on $(X, E)$ and $(F, E) \widetilde{\subseteq}(X, E)$ be a soft set. The soft interior of $(F, E)$ on $\widetilde{\varphi}($ denoted by $\widetilde{\imath}_{\widetilde{\varphi}}((F, E))$ is defined as follows:

$$
\widetilde{\boldsymbol{l}}_{\widetilde{\varphi}}((F, E))=\left\{x_{e} \widetilde{\in}(F, E):(V, E) \in \widetilde{\varphi}\left(x_{e}\right) \text { such that }(V, E) \widetilde{\widetilde{C}}(F, E)\right\} .
$$

Theorem 1. Let $(X, \widetilde{\varphi}, E)$ be a WSNS on $(X, E)$. Then the following conditions are satisfied:

(i) For all $(F, E) \widetilde{\subseteq}(X, E), \widetilde{\imath}_{\widetilde{\varphi}}((F, E)) \widetilde{\subseteq}(F, E)$,

(ii) For all $(F, E),(G, E) \widetilde{\subseteq}(X, E), \widetilde{\imath}_{\widetilde{\varphi}}((F, E) \widetilde{\cap}(G, E))=\widetilde{\imath}_{\widetilde{\varphi}}((F, E)) \widetilde{\cap} \widetilde{\imath}_{\widetilde{\varphi}}((G, E))$,

(iii) $\widetilde{\imath}_{\widetilde{\varphi}}((X, E))=(X, E)$.

Proof. (1) and (3) are straighforward.

(2) Let $x_{e} \widetilde{\in}_{\widetilde{\imath}}((F, E)) \widetilde{\cap} \widetilde{\imath}_{\widetilde{\varphi}}((G, E))$. Then there are two weak soft neighborhood $(U, E),(V, E) \in \widetilde{\varphi}\left(x_{e}\right)$ such that $(U, E) \widetilde{\widetilde{\subseteq}}(F, E)$ and $(V, E) \widetilde{\widetilde{\subseteq}}(G, E)$. From the Definition 25.(ii), we have $(U, E) \widetilde{\cap}(V, E) \in \widetilde{\varphi}\left(x_{e}\right)$. Therefore, $x_{e} \widetilde{\imath}_{\widetilde{\varphi}}((F, E) \widetilde{\cap}(G, E))$.

The convere of proof is obvious.

Example 1. Let $X=\left\{x^{1}, x^{2}, x^{3}\right\}, E=\left\{e_{1}, e_{2}\right\}$ and $\widetilde{\varphi}: S S(X)_{E} \rightarrow P\left(S S(X)_{E}\right)$ be a weak soft neighborhood system defined as follows:

$$
\begin{aligned}
& \widetilde{\varphi}\left(x_{e_{1}}^{1}\right)=\left[\left\{\left(e_{1},\left\{x^{1}, x^{2}\right\}\right),\left(e_{2},\left\{x^{2}\right\}\right)\right\}\right], \\
& \widetilde{\varphi}\left(x_{e_{2}}^{1}\right)=\left[\left\{\left(e_{1},\left\{x^{1}\right\}\right),\left(e_{2},\left\{x^{1}, x^{2}\right\}\right)\right\}\right], \\
& \widetilde{\varphi}\left(x_{e_{1}}^{2}\right)=\left[\left\{\left(e_{1},\left\{x^{2}, x^{3}\right\}\right),\left(e_{2},\left\{x^{1}\right\}\right)\right\}\right], \\
& \widetilde{\varphi}\left(x_{e_{2}}^{2}\right)=\left[\left\{\left(e_{1},\left\{x^{1}, x^{3}\right\}\right),\left(e_{2},\left\{x^{2}\right\}\right)\right\}\right], \\
& \widetilde{\varphi}\left(x_{e_{1}}^{3}\right)=\left[\left\{\left(e_{1},\left\{x^{1}, x^{3}\right\}\right),\left(e_{2},\left\{x^{1}, x^{2}\right\}\right)\right\}\right], \\
& \widetilde{\varphi}\left(x_{e_{2}}^{3}\right)=[\widetilde{X}] .
\end{aligned}
$$


Suppose that $(F, E)=\left\{\left(e_{1},\left\{x^{1}, x^{2}\right\}\right),\left(e_{2},\left\{x^{1}, x^{2}, x^{3}\right\}\right)\right\} \widetilde{\subseteq}(X, E)$. Then $\widetilde{\imath}_{\widetilde{\varphi}}((F, E))=\left\{\left(e_{1},\left\{x^{1}\right\}\right),\left(e_{2},\left\{x^{1}\right\}\right)\right\}$ but $\widetilde{\imath}_{\widetilde{\varphi}}\left(\widetilde{l}_{\widetilde{\varphi}}((F, E))\right)=\Phi$ and so $\widetilde{l}_{\widetilde{\varphi}}((F, E)) \neq \widetilde{l}_{\widetilde{\varphi}}\left(\widetilde{l}_{\tilde{\varphi}}((F, E))\right)$.

Definition 27. Let $(X, \widetilde{\varphi}, E)$ be a WSNS on $(X, E)$ and $(F, E) \widetilde{\subseteq}(X, E)$ be a soft set. The soft closure of $(F, E)$ on $\widetilde{\varphi}$ (denoted by $\widetilde{\kappa}_{\tilde{\varphi}}((F, E))$ is defined as follows:

$$
\widetilde{\kappa}_{\widetilde{\varphi}}((F, E))=\left\{x_{e} \widetilde{\in} S S(X)_{E}:(V, E) \widetilde{\cap}(F, E) \neq \Phi \text { for all }(V, E) \in \widetilde{\varphi}\left(x_{e}\right)\right\} .
$$

Example 2. According to the Example 1, The soft closure $\widetilde{\kappa}_{\widetilde{\varphi}}((F, E))=\widetilde{X}$.

Theorem 2. Let $(X, \widetilde{\varphi}, E)$ be a WSNS on $(X, E)$. Then the following conditions are satisfied:

(i) $\widetilde{\kappa}_{\widetilde{\varphi}}(\Phi)=\Phi$,

(ii) For all $(F, E) \widetilde{\subseteq}(X, E),(F, E) \widetilde{\subseteq} \widetilde{\kappa}_{\widetilde{\varphi}}((F, E))$,

(iii) For all $(F, E),(G, E) \widetilde{\subseteq}(X, E), \widetilde{\kappa}_{\widetilde{\varphi}}((F, E) \widetilde{\cup}(G, E))=\widetilde{\kappa}_{\widetilde{\varphi}}((F, E)) \widetilde{\cup} \widetilde{\kappa}_{\tilde{\varphi}}((G, E))$,

(iv) $\widetilde{\kappa}_{\widetilde{\varphi}}((F, E))=\left(\widetilde{\boldsymbol{l}}_{\widetilde{\varphi}}\left((F, E)^{c}\right)\right)^{c}, \widetilde{\boldsymbol{l}}_{\widetilde{\varphi}}((F, E))=\left(\widetilde{\kappa}_{\widetilde{\varphi}}\left((F, E)^{c}\right)\right)^{c}$.

Proof. From the Definition 27. and the Definition 26. it is obvious.

Definition 28. A mapping $\widetilde{J}: S S(X)_{E} \rightarrow S S(X)_{E}$ is said to be a soft interior operator on the soft set $(X, E)$ if the following conditions hold.

(1) For all $(F, E) \widetilde{\subseteq}(X, E), \widetilde{J}((F, E)) \widetilde{\subseteq}(F, E)$,

(2) For all $(F, E),(G, E) \widetilde{\subseteq}(X, E), \widetilde{J}((F, E) \widetilde{\cap}(G, E))=\widetilde{J}((F, E)) \widetilde{\cap} \widetilde{J}((G, E))$,

(3) $\widetilde{J}((X, E))=(X, E)$.

From the Theorem 1. and the definition of soft interior operator, we have the following results.

\section{Theorem 3.}

(1) Let $(X, \widetilde{\varphi}, E)$ be a WSNS on $(X, E)$ and $\widetilde{J}: S S(X)_{E} \rightarrow S S(X)_{E}$ be defined as $\widetilde{J}((F, E))=\widetilde{\boldsymbol{\tau}}_{\widetilde{\varphi}}((F, E))$ for each $(F, E) \widetilde{\subseteq}(X, E)$. Then $\widetilde{J}$ is a soft interior operator.

(2) Let $\widetilde{J}: S S(X)_{E} \rightarrow S S(X)_{E}$ be a soft interior operator and $\widetilde{\theta}_{\widetilde{J}}: S S(X)_{E} \rightarrow P\left(S S(X)_{E}\right)$ be defined as $\widetilde{\theta}_{\widetilde{J}}\left(x_{e}\right)=\left\{\widetilde{J}((F, E)): x_{e} \widetilde{\in} \widetilde{J}((F, E))\right.$ for $\left.(F, E) \widetilde{\subseteq}(X, E)\right\}$. Then $\widetilde{\theta}_{\widetilde{J}}$ is a weak soft neighborhood system induced by soft interior operator $\widetilde{J}$.

Let $(X, \widetilde{\varphi}, E)$ be a WSNS on $(X, E)$ and $\widetilde{J}: S S(X)_{E} \rightarrow S S(X)_{E}$ be defined as $\widetilde{J}((F, E))=\widetilde{\imath}_{\widetilde{\varphi}}((F, E))$ for each $(F, E) \widetilde{\subseteq}(X, E)$. Then the soft interior operator $\widetilde{J}=\widetilde{l}_{\widetilde{\varphi}}$ induces a weak soft neighborhood system $\widetilde{\theta}_{i_{\tilde{\varphi}}}$. Moreover, $\widetilde{\theta}_{\tau_{\widetilde{\varphi}}}$ and $\widetilde{\varphi}$ are not equal as the following example shows.

Example 3. Let $X=\left\{x^{1}, x^{2}, x^{3}\right\}, E=\{e\}$ and $\widetilde{\varphi}: S S(X)_{E} \rightarrow P\left(S S(X)_{E}\right)$ be a weak soft neighborhood system defined as follows:

$$
\begin{aligned}
& \widetilde{\varphi}\left(x_{e}^{1}\right)=\left[\left\{\left(e,\left\{x^{1}\right\}\right)\right\},\left\{\left(e,\left\{x^{1}, x^{3}\right\}\right)\right\}\right], \\
& \widetilde{\varphi}\left(x_{e}^{2}\right)=\left[\left\{\left(e,\left\{x^{2}\right\}\right)\right\},\left\{\left(e,\left\{x^{2}, x^{3}\right\}\right)\right\}\right], \\
& \widetilde{\varphi}\left(x_{e}^{3}\right)=[\widetilde{X}] .
\end{aligned}
$$

Then $\widetilde{\theta}_{\tilde{l}_{\tilde{\varphi}}}$ is a weak soft neighborhood system induced by $\widetilde{\boldsymbol{l}}_{\widetilde{\varphi}}$ as follows:

$$
\begin{aligned}
& \widetilde{\theta}_{\tilde{l}_{\tilde{\varphi}}}\left(x_{e}^{1}\right)=\left[\left\{\left(e,\left\{x^{1}\right\}\right)\right\},\left\{\left(e,\left\{x^{1}, x^{2}\right\}\right)\right\}, \widetilde{X}\right], \\
& \widetilde{\theta}_{\tau_{\tilde{\varphi}}}\left(x_{e}^{2}\right)=\left[\left\{\left(e,\left\{x^{2}\right\}\right)\right\},\left\{\left(e,\left\{x^{1}, x^{2}\right\}\right)\right\}, \widetilde{X}\right], \\
& \widetilde{\theta}_{l_{\tilde{\varphi}}}\left(x_{e}^{3}\right)=[\widetilde{X}] .
\end{aligned}
$$


Definition 29. Let $(X, \widetilde{\varphi}, E),\left(Y, \widetilde{\varphi}^{\prime}, E^{\prime}\right)$ be two WSNS's and $(f, g):(X, \widetilde{\varphi}, E) \rightarrow\left(Y, \widetilde{\varphi}^{\prime}, E^{\prime}\right)$ be a soft mapping. Then $(f, g)$ is called soft $w^{s}\left(\widetilde{\varphi}, \widetilde{\varphi}^{\prime}\right)$ - continuous if there is $(U, E) \in \widetilde{\varphi}\left(x_{e}\right)$ such that $(f, g)((U, E)) \widetilde{\subseteq}\left(V, E^{\prime}\right)$ for $x_{e} \widetilde{\in} S S(X)_{E}$ and $\left(V, E^{\prime}\right) \in \widetilde{\varphi}^{\prime}\left(f(x)_{g(e)}\right)$.

Theorem 4. Let $(f, g):(X, \widetilde{\varphi}, E) \rightarrow\left(Y, \widetilde{\varphi}^{\prime}, E^{\prime}\right)$ be a soft mapping on two WSNS's $(X, \widetilde{\varphi}, E)$ and $\left(Y, \widetilde{\varphi}^{\prime}, E^{\prime}\right)$. Then the following statements are equivalent:

(1) $(f, g)$ is soft $w^{s}\left(\widetilde{\varphi}, \widetilde{\varphi}^{\prime}\right)-$ continuous,

(2) $\operatorname{For}(F, E) \widetilde{\subseteq}(X, E),(f, g)\left(\widetilde{\kappa}_{\widetilde{\varphi}}((F, E))\right) \widetilde{\subseteq} \widetilde{\kappa}_{\widetilde{\varphi}^{\prime}}((f, g)((F, E)))$,

(3) $\operatorname{For}\left(G, E^{\prime}\right) \widetilde{\subseteq}\left(Y, E^{\prime}\right), \widetilde{\kappa}_{\widetilde{\varphi}}\left((f, g)^{-1}\left(\left(G, E^{\prime}\right)\right)\right) \widetilde{\subseteq}(f, g)^{-1}\left(\widetilde{\kappa}_{\widetilde{\varphi}^{\prime}}\left(\left(G, E^{\prime}\right)\right)\right)$,

(4) $\operatorname{For}\left(G, E^{\prime}\right) \widetilde{\subseteq}\left(Y, E^{\prime}\right),(f, g)^{-1}\left(\widetilde{\imath}_{\widetilde{\varphi}^{\prime}}\left(\left(G, E^{\prime}\right)\right)\right) \widetilde{\widetilde{c}} \widetilde{\imath}_{\widetilde{\varphi}}\left((f, g)^{-1}\left(\left(G, E^{\prime}\right)\right)\right)$.

Proof. (1) $\Rightarrow(2)$ Let $x_{e} \widetilde{\in} \widetilde{\kappa}_{\widetilde{\varphi}}((F, E))$. Suppose that $f(x)_{g(e)} \widetilde{\notin}_{\widetilde{\boldsymbol{\varphi}}_{\widetilde{\varphi}^{\prime}}}((f, g)((F, E)))$. Then there exists $\left(V, E^{\prime}\right) \in \widetilde{\varphi}^{\prime}\left(f(x)_{g(e)}\right)$ such that $\left(V, E^{\prime}\right) \widetilde{\cap} \widetilde{\kappa}_{\widetilde{\varphi}^{\prime}}((f, g)((F, E)))=\Phi$. From the soft $w^{s}\left(\widetilde{\varphi}, \widetilde{\varphi}^{\prime}\right)-$ continuity, there exists $(U, E) \in \widetilde{\varphi}\left(x_{e}\right)$ such that $(f, g)((U, E)) \widetilde{\subseteq}\left(V, E^{\prime}\right)$, and so

$$
(f, g)((U, E)) \widetilde{\cap} \widetilde{\kappa}_{\widetilde{\varphi}^{\prime}}((f, g)((F, E)))=\Phi .
$$

Therefore, $(U, E) \widetilde{\cap}(F, E)=\Phi$; This is a contradiction. That is, $f(x)_{g(e)} \widetilde{\in}_{\widetilde{\kappa}_{\tilde{\varphi}^{\prime}}}((f, g)((F, E)))$. $(2) \Rightarrow(3)$ Suppose that $(F, E)=(f, g)^{-1}\left(\left(G, E^{\prime}\right)\right)$ for $\left(G, E^{\prime}\right) \widetilde{\subseteq}\left(Y, E^{\prime}\right)$. From the hypothesis,

$$
(f, g)\left(\widetilde{\kappa}_{\widetilde{\varphi}}((F, E))\right) \widetilde{\subseteq} \widetilde{\kappa}_{\widetilde{\varphi}^{\prime}}((f, g)((F, E)))=\widetilde{\kappa}_{\widetilde{\varphi}^{\prime}}(f, g)\left((f, g)^{-1}\left(\left(G, E^{\prime}\right)\right)\right) \widetilde{\subseteq} \widetilde{\kappa}_{\widetilde{\varphi}^{\prime}}\left(\left(G, E^{\prime}\right)\right)
$$

Thus, $\widetilde{\kappa}_{\widetilde{\varphi}}\left((f, g)^{-1}\left(\left(G, E^{\prime}\right)\right)\right) \widetilde{\subseteq}(f, g)^{-1}\left(\widetilde{\kappa}_{\widetilde{\varphi}^{\prime}}\left(\left(G, E^{\prime}\right)\right)\right)$.

$(3) \Rightarrow(4)$ From the Theorem 2, it is obvious.

$(4) \Rightarrow(1)$ Let $\left(V, E^{\prime}\right) \in \widetilde{\varphi}^{\prime}\left(f(x)_{g(e)}\right)$ for $x_{e} \widetilde{\in} S S(X)_{E}$. Then $f(x)_{g(e)} \widetilde{\tilde{l}}_{\widetilde{\varphi}^{\prime}}\left(\left(V, E^{\prime}\right)\right)$. From the hypothesis, $x_{e} \widetilde{\in}(f, g)^{-1}\left(\widetilde{\boldsymbol{l}}_{\widetilde{\varphi}^{\prime}}\left(\left(V, E^{\prime}\right)\right)\right) \widetilde{\widetilde{\imath}} \widetilde{\tau}_{\widetilde{\varphi}}\left((f, g)^{-1}\left(\left(V, E^{\prime}\right)\right)\right)$. By the definition of soft interior, there exists $(U, E) \in \widetilde{\varphi}\left(x_{e}\right)$ such that $(U, E) \widetilde{\subseteq}(f, g)^{-1}\left(\left(V, E^{\prime}\right)\right)$. Therefore, $(f, g)$ is soft $w^{s}\left(\widetilde{\varphi}, \widetilde{\varphi}^{\prime}\right)$-continuous.

Definition 30. Let $(X, \widetilde{\varphi}, E)$ be a WSNS on $(X, E)$ and $(F, E) \widetilde{\subseteq}(X, E)$ be a soft set. Then $(F, E)$ is called a soft $w_{\widetilde{\varphi}}^{s}-$ open set if there exists $(V, E) \in \widetilde{\varphi}\left(x_{e}\right)$ such that $(V, E) \widetilde{\subseteq}(F, E)$ for each $x_{e} \widetilde{\in}(F, E)$.

Let $W S_{\widetilde{\varphi}}(X)_{E}$ denote the collection of all soft $w_{\widetilde{\varphi}}^{s}-$ open set on a a WSNS $(X, \widetilde{\varphi}, E)$. The complements of soft $w_{\widetilde{\varphi}}^{s}-$ open sets are called soft $w_{\widetilde{\varphi}}^{s}-$ closed sets.

Theorem 5. Let $(X, \widetilde{\varphi}, E)$ be a WSNS on $(X, E)$. Then the collection $W S_{\widetilde{\varphi}}(X)_{E}$ of all soft $w_{\widetilde{\varphi}}^{s}-$ open subsets of $(X, E)$ is a soft topology on $(X, E)$.

Proof. (1) It is clear that $\Phi$ and $\widetilde{X}$ are soft $w_{\widetilde{\varphi}}^{s}$-open sets.

(2) Let $(F, E)$ and $(G, E)$ be two soft $w_{\widetilde{\varphi}}^{s}-$ open sets. Then for each $x_{e} \widetilde{\in}(F, E) \widetilde{\cap}(G, E)$ there exists $(U, E),(V, E) \in \widetilde{\varphi}\left(x_{e}\right)$ such that $(U, E) \widetilde{\subseteq}(F, E),(V, E) \widetilde{\subseteq}(G, E)$. Since $\widetilde{\varphi}\left(x_{e}\right)$ is a weak soft neighborhood, then $(U, E) \widetilde{\cap}(V, E) \in \widetilde{\varphi}\left(x_{e}\right)$. Therefore, $(F, E) \widetilde{\cap}(G, E)$ is also soft $w_{\widetilde{\varphi}}^{s}-$ open set. 
(3) Let $\left(F_{i}, E\right)_{i \in I} \in W S_{\widetilde{\varphi}}(X)_{E}$. In this case, for each $x_{e} \widetilde{\in} \widetilde{\cup}_{i \in I}\left(F_{i}, E\right)$, there exists $\left(F_{i}, E\right)$ such that $x_{e} \widetilde{\in}\left(F_{i}, E\right)$ for $i \in I$, and so there is $(V, E) \in \widetilde{\varphi}\left(x_{e}\right)$ such that $(V, E) \widetilde{\subseteq}\left(F_{i}, E\right) \widetilde{\subseteq} \widetilde{\widetilde{U}}\left(F_{i}, E\right)$. Thus, $\underset{i \in I}{\widetilde{U}}\left(F_{i}, E\right)$ is a soft $w_{\widetilde{\varphi}}^{s}-$ open set.

Remark. Let us define $\widetilde{\varphi}: S S(X)_{E} \rightarrow P\left(S S(X)_{E}\right)$ in a soft topological space $(X, \widetilde{\tau}, E)$ as

$$
\widetilde{\varphi}\left(x_{e}\right)=\left\{(F, E) \in \widetilde{\tau}: x_{e} \widetilde{\in}(F, E)\right\}
$$

for $x_{e} \widetilde{\in} S S(X)_{E}$. Then $\widetilde{\varphi}$ is a weak soft neighborhood system. Thus, $W S_{\widetilde{\varphi}}(X)_{E}=\widetilde{\tau}$.

Definition 31. Let $(X, \widetilde{\varphi}, E)$ be a WSNS on $(X, E)$ and $(F, E) \widetilde{\subseteq}(X, E)$ be a soft set. The soft $w_{\widetilde{\varphi}}^{s}$-interior of $(F, E)$ (denoted by $\left.\widetilde{i}_{\widetilde{\varphi}}((F, E))\right)$ is the soft union of all $(G, E) \widetilde{\subseteq}(F, E)$ such that $(G, E) \in W S_{\widetilde{\varphi}}(X)_{E}$. The soft $w_{\widetilde{\varphi}}^{s}$-closure of $(F, E)$ (denoted by $\left.\widetilde{c}_{\tilde{\varphi}}((F, E))\right)$ is the soft intersection of all soft $w_{\tilde{\varphi}}^{s}$-closed sets containing $(F, E)$.

Theorem 6. Let $(X, \widetilde{\varphi}, E)$ be a WSNS on $(X, E)$ and $(F, E) \widetilde{\subseteq}(X, E)$ be a soft set. Then the following conditions are satisfied.

(1) $\widetilde{i}_{\widetilde{\varphi}}((F, E)) \widetilde{\widetilde{c}} \widetilde{\boldsymbol{l}}_{\widetilde{\varphi}}((F, E))$,

(2) $\widetilde{\kappa}_{\widetilde{\varphi}}((F, E)) \widetilde{\subseteq} \widetilde{c}_{\widetilde{\varphi}}((F, E))$,

(3) $(F, E)$ is soft $w_{\widetilde{\varphi}}^{s}$ - open set iff $\widetilde{i}_{\widetilde{\varphi}}((F, E))=(F, E)$,

(4) $(F, E)$ is soft $w_{\widetilde{\varphi}}^{s}-$ closed set iff $\widetilde{c}_{\widetilde{\varphi}}((F, E))=(F, E)$.

Proof. (1) Let $x_{e} \widetilde{\in} \tilde{i}_{\widetilde{\varphi}}((F, E))$. Then there exists a soft $w_{\widetilde{\varphi}}^{s}-$ open set $(G, E)$ such that $x_{e} \widetilde{\in}(G, E) \widetilde{\subseteq}(F, E)$. From the definition of soft $w_{\widetilde{\varphi}}^{s}-$ open set, there exists $(V, E) \in \widetilde{\varphi}\left(x_{e}\right)$ such that $x_{e} \widetilde{\in}(V, E) \widetilde{\subseteq}(G, E) \widetilde{\subseteq}(F, E)$. Therefore, $x_{e} \widetilde{\in} \widetilde{l}_{\widetilde{\varphi}}((F, E))$.

(2) It is similar to (1).

(3) and (4) are straighforward.

Example 4. Let $X=\left\{x^{1}, x^{2}, x^{3}\right\}, E=\left\{e_{1}, e_{2}\right\}$ and $\widetilde{\varphi}: S S(X)_{E} \rightarrow P\left(S S(X)_{E}\right)$ be a weak soft neighborhood system defined as follows:

$$
\begin{aligned}
& \widetilde{\varphi}\left(x_{e_{1}}^{1}\right)=\left[\left\{\left(e_{1},\left\{x^{1}, x^{2}\right\}\right),\left(e_{2},\left\{x^{2}\right\}\right)\right\}\right], \\
& \widetilde{\varphi}\left(x_{e_{2}}^{1}\right)=\left[\left\{\left(e_{1},\left\{x^{3}\right\}\right),\left(e_{2},\left\{x^{1}, x^{3}\right\}\right)\right\}\right], \\
& \widetilde{\varphi}\left(x_{e_{1}}^{2}\right)=\left[\left\{\left(e_{1},\left\{x^{1}, x^{2}\right\}\right),\left(e_{2},\left\{x^{2}, x^{3}\right\}\right)\right\}\right], \\
& \widetilde{\varphi}\left(x_{e_{2}}^{2}\right)=\left[\left\{\left(e_{1},\left\{x^{1}\right\}\right),\left(e_{2},\left\{x^{2}\right\}\right)\right\}\right], \\
& \widetilde{\varphi}\left(x_{e_{1}}^{3}\right)=\left[\left\{\left(e_{1},\left\{x^{3}\right\}\right),\left(e_{2},\left\{x^{1}, x^{2}, x^{3}\right\}\right)\right\}\right], \\
& \widetilde{\varphi}\left(x_{e_{2}}^{3}\right)=\left[\left\{\left(e_{1}, \emptyset\right),\left(e_{2},\left\{x^{2}, x^{3}\right\}\right)\right\}\right] .
\end{aligned}
$$

Suppose that

$$
(F, E)=\left\{\left(e_{1},\left\{x^{1}, x^{2}\right\}\right),\left(e_{2},\left\{x^{1}, x^{2}, x^{3}\right\}\right)\right\} \widetilde{\subseteq}(X, E) .
$$

Then

$$
\widetilde{\boldsymbol{l}}_{\widetilde{\varphi}}((F, E))=\left\{\left(e_{1},\left\{x^{1}, x^{2}\right\}\right),\left(e_{2},\left\{x^{2}, x^{3}\right\}\right)\right\}
$$

and

$$
\widetilde{i}_{\widetilde{\varphi}}((F, E))=\left\{\left(e_{1},\left\{x^{2}\right\}\right),\left(e_{2}, \emptyset\right)\right\} .
$$

Thus,

$$
\widetilde{\boldsymbol{i}}_{\widetilde{\varphi}}((F, E)) \neq \widetilde{i}_{\widetilde{\varphi}}((F, E)) .
$$


Theorem 7. Let $(X, \widetilde{\varphi}, E)$ be a WSNS on $(X, E)$ and $(F, E) \widetilde{\subseteq}(X, E)$ be a soft set. Then $\widetilde{\imath}_{\widetilde{\varphi}}((F, E))=(F, E)$ iff $(F, E)$ is soft $w_{\widetilde{\varphi}}^{s}$ open set.

Proof. Let $\widetilde{\boldsymbol{l}}_{\widetilde{\varphi}}((F, E))=(F, E)$. There exists $(V, E) \in \widetilde{\varphi}\left(x_{e}\right)$ such that $x_{e} \widetilde{\in}(V, E) \widetilde{\subseteq}(F, E)=\widetilde{\boldsymbol{l}}_{\widetilde{\varphi}}((F, E))$, and so $\widetilde{\boldsymbol{l}}_{\widetilde{\varphi}}((F, E))$ is a soft $w_{\widetilde{\varphi}}^{s}-$ open set.

Conversely, suppose that $(F, E)$ is soft $w_{\widetilde{\varphi}}^{s}-$ open set. From the Theorem 6.(3), We have

$$
(F, E)=\widetilde{i}_{\widetilde{\varphi}}((F, E)) \widetilde{\widetilde{\complement}} \widetilde{l}_{\widetilde{\varphi}}((F, E)) \widetilde{\simeq}(F, E) .
$$

That is, $\widetilde{\imath}_{\widetilde{\varphi}}((F, E))=(F, E)$.

Theorem 8. Let $\widetilde{J}: S S(X)_{E} \rightarrow S S(X)_{E}$ be a soft interior operator. Then there exists a weak soft neighborhood system $\widetilde{\varphi}$ induced by $\widetilde{J}$ such that $(F, E)$ is soft $w_{\widetilde{\varphi}}^{s}-$ open set iff $\widetilde{J}((F, E))=(F, E)$.

Proof. Let us define $\widetilde{\varphi}: S S(X)_{E} \rightarrow P\left(S S(X)_{E}\right)$ by $\widetilde{\varphi}\left(x_{e}\right)=\left\{(V, E): \widetilde{J}((V, E))=(V, E)\right.$ and $\left.x_{e} \widetilde{\in}(V, E)\right\}$ for $x_{e} \widetilde{\in} S S(X)_{E}$. Then $\widetilde{\varphi}$ is a WSNS. $(F, E)$ is soft $w_{\widetilde{\varphi}}^{s}-$ open set iff $(F, E)=\underset{x_{e}}{\widetilde{U}(F, E)}(V, E)$ where $(V, E) \in \widetilde{\varphi}\left(x_{e}\right)$. Since $\widetilde{J}((V, E))=(V, E)$ for $(V, E) \in \widetilde{\varphi}\left(x_{e}\right)$, then

$$
(F, E)=\underset{x_{e} \widetilde{\in}(F, E)}{\widetilde{U}}(V, E)=\underset{x_{e}}{\widetilde{U}(F, E)} \widetilde{J}((V, E)) \widetilde{\subseteq} \widetilde{J}\left(\underset{x_{e} \widetilde{\epsilon}(F, E)}{\widetilde{U}}(V, E)\right)=\widetilde{J}((F, E)) .
$$

Therefore, $(F, E)$ is a soft $w_{\widetilde{\varphi}}^{s}-$ open set iff $\widetilde{J}((F, E))=(F, E)$.

Definition 32. Let $(X, \widetilde{\varphi}, E),\left(Y, \widetilde{\varphi}^{\prime}, E^{\prime}\right)$ be two WSNS's and $(f, g):(X, \widetilde{\varphi}, E) \rightarrow\left(Y, \widetilde{\varphi}^{\prime}, E^{\prime}\right)$ be a soft mapping. Then $(f, g)$ is called soft $w^{s}$ - continuous if $(f, g)^{-1}((G, E))$ is soft $w_{\widetilde{\varphi}}^{s}$-open set in $W S_{\widetilde{\varphi}}(X)_{E}$ for every soft $w_{\widetilde{\varphi}^{\prime}}^{s}-$ open set $(G, E) \in$ $W S_{\widetilde{\varphi}^{\prime}}(Y)_{E^{\prime}}$

Theorem 9. Let $(f, g):(X, \widetilde{\varphi}, E) \rightarrow\left(Y, \widetilde{\varphi}^{\prime}, E^{\prime}\right)$ be a soft mapping on two WSNS's $(X, \widetilde{\varphi}, E)$ and $\left(Y, \widetilde{\varphi}^{\prime}, E^{\prime}\right)$. Then the following statements are equivalent:

(1) $(f, g)$ is soft $w^{s}-$ continuous;

(2) For each $w_{\widetilde{\varphi}^{\prime}}^{s}-$ closed set $(G, E)$ in $\left(Y, E^{\prime}\right),(f, g)^{-1}((G, E))$ is soft $w_{\widetilde{\varphi}}^{s}-$ closed set in $(X, E)$,

(3) For all $(F, E) \widetilde{\subseteq}(X, E),(f, g)\left(\widetilde{c}_{\widetilde{\varphi}}((F, E))\right) \widetilde{\widetilde{\subseteq}} \widetilde{c}_{\widetilde{\varphi}^{\prime}}((f, g)((F, E)))$,

(4) For all $(G, E) \widetilde{\subseteq}(Y, E), \widetilde{c}_{\widetilde{\varphi}}\left((f, g)^{-1}((G, E))\right) \widetilde{\subseteq}(f, g)^{-1}\left(\widetilde{c}_{\widetilde{\varphi}^{\prime}}((G, E))\right)$,

(5) For all $(G, E) \widetilde{\subseteq}(Y, E),(f, g)^{-1}\left(\widetilde{i}_{\widetilde{\varphi}^{\prime}}((G, E))\right) \widetilde{\subseteq} \widetilde{i_{\breve{\varphi}}}\left((f, g)^{-1}((G, E))\right)$.

Proof. Straighforward.

Theorem 10. Let $(f, g):(X, \widetilde{\varphi}, E) \rightarrow\left(Y, \widetilde{\varphi}^{\prime}, E^{\prime}\right)$ be a soft mapping on two WSNS's $(X, \widetilde{\varphi}, E)$ and $\left(Y, \widetilde{\varphi}^{\prime}, E^{\prime}\right)$. If $(f, g)$ is soft $w^{s}\left(\widetilde{\varphi}, \widetilde{\varphi}^{\prime}\right)$-continuous, then it is also soft $w^{s}-$ continuous.

Proof. Let $(G, E)$ be a soft $w_{\widetilde{\varphi}^{\prime}}^{s}-$ open set. From the Theorem 7, it follows $\widetilde{\boldsymbol{l}}_{\widetilde{\varphi}}((G, E))=(G, E)$. By Theorem 3.7. and Theorem 3.10.(5), we have

$$
(f, g)^{-1}((G, E))=(f, g)^{-1}\left(\widetilde{\imath}_{\widetilde{\varphi}}((G, E))\right) \widetilde{\widetilde{\complement}} \widetilde{\imath}_{\widetilde{\varphi}}\left((f, g)^{-1}((G, E))\right)
$$

Therefore, $(f, g)^{-1}((G, E))$ is $w_{\widetilde{\varphi}}^{s}$-open set.

From the following example, the converse of Theorem 10 is not true. 
Example 5. Let $X=\left\{x^{1}, x^{2}, x^{3}, x^{4}\right\}, E=\{e\}$ and $\widetilde{\varphi}: S S(X)_{E} \rightarrow P\left(S S(X)_{E}\right)$ be a weak soft neighborhood system defined as follows:

$$
\begin{aligned}
& \widetilde{\varphi}\left(x_{e}^{1}\right)=\left[\left\{\left(e,\left\{x^{1}, x^{3}\right\}\right)\right\}\right], \\
& \widetilde{\varphi}\left(x_{e}^{2}\right)=\left[\left\{\left(e,\left\{x^{2}\right\}\right)\right\}\right], \\
& \widetilde{\varphi}\left(x_{e}^{3}\right)=\widetilde{\varphi}\left(x_{e}^{4}\right)=[\widetilde{X}] .
\end{aligned}
$$

Let us define the soft mapping $\left(f, 1_{E}\right):\left(X, W S_{\widetilde{\varphi}}(X)_{E}, E\right) \rightarrow\left(X, W S_{\widetilde{\varphi}}(X)_{E}, E\right)$ as follows:

$$
\begin{aligned}
& f\left(x^{1}\right)_{1_{E}(e)}=x_{e}^{1}, f\left(x^{2}\right)_{1_{E}(e)}=x_{e}^{2}, \\
& f\left(x^{3}\right)_{1_{E}(e)}=x_{e}^{4}, f\left(x^{4}\right)_{1_{E}(e)}=x_{e}^{3} .
\end{aligned}
$$

Then $\left(f, 1_{E}\right)$ is soft $w^{s}$-continuous, but it is not soft $w^{s}(\widetilde{\varphi}, \widetilde{\varphi})-$ continuous because for $x_{e}^{1} \widetilde{\in} S S(X)_{E}$ and $(V, E)=\left\{\left(e,\left\{x^{1}, x^{3}\right\}\right)\right\} \in \widetilde{\varphi}\left(f\left(x^{1}\right)_{1_{E}(e)}\right)$, there is no $(U, E) \in \widetilde{\varphi}\left(x_{e}^{1}\right)$ such that $\left(f, 1_{E}\right)((U, E)) \widetilde{\subseteq}(V, E)$.

\section{New soft interior and closure operators on a WSNS}

Definition 33. Let $(X, \widetilde{\varphi}, E)$ be a WSNS on $(X, E)$ and $(F, E) \widetilde{\subseteq}(X, E)$ be a soft set. Then,

(1) $\widetilde{J_{\widetilde{\varphi}}^{*}}((F, E))=\left\{x_{e} \widetilde{\in}(F, E):(F, E) \in \widetilde{\varphi}\left(x_{e}\right)\right\}$,

(2) $\widetilde{c}_{\widetilde{\varphi}}^{*}((F, E))=\left\{x_{e} \widetilde{\in}(X, E):(F, E)^{c} \notin \widetilde{\varphi}\left(x_{e}\right)\right\}$.

Theorem 11. Let $(X, \widetilde{\varphi}, E)$ be a WSNS on $(X, E)$ and $(F, E),(G, E) \widetilde{\subseteq}(X, E)$ be two soft sets. Then the following statements are satisfied.

(1) $\widetilde{J}_{\widetilde{\varphi}}^{*}((F, E)) \widetilde{\subseteq}(F, E)$,

(2) $\widetilde{J_{\widetilde{\varphi}}^{*}}((F, E)) \widetilde{\cap} \widetilde{J}_{\widetilde{\varphi}}^{*}((G, E)) \widetilde{\subseteq} \widetilde{J}_{\widetilde{\varphi}}^{*}((F, E) \widetilde{\cap}(G, E))$,

(3) $\widetilde{J_{\widetilde{\varphi}}^{*}}((F, E)) \widetilde{\widetilde{\subseteq}} \widetilde{\varphi} \widetilde{\varphi}((F, E))$,

(4) $\widetilde{J}_{\widetilde{\varphi}}^{*}((F, E))=\left(\widetilde{c}_{\widetilde{\varphi}}^{*}\left((F, E)^{c}\right)\right)^{c}$.

Proof.(1) Straighforward.

(2) Let $x_{e} \widetilde{\in} \widetilde{J_{\tilde{\varphi}}^{*}}((F, E)) \widetilde{\cap} \widetilde{J}_{\widetilde{\varphi}}^{*}((G, E))$. Then $(F, E)$ and $(G, E) \in \widetilde{\varphi}\left(x_{e}\right)$. From the property of weak soft neighborhood, $(F, E) \widetilde{\cap}(G, E) \in \widetilde{\varphi}\left(x_{e}\right)$. Therefore, $x_{e} \widetilde{\in} \widetilde{J}_{\widetilde{\varphi}}^{*}((F, E) \widetilde{\cap}(G, E))$.

(3) Obvious.

(4) Let $x_{e} \widetilde{\in} \widetilde{J}_{\widetilde{\varphi}}^{*}((F, E))$ for $(F, E) \widetilde{\subseteq}(X, E)$. In this case, $(F, E)=\left((F, E)^{c}\right)^{c} \in \widetilde{\varphi}\left(x_{e}\right)$ and by Definition 33, $x_{e} \widetilde{\notin} \widetilde{c_{\tilde{\varphi}}}\left((F, E)^{c}\right)$. Hence, we have $x_{e} \widetilde{\in}\left(\widetilde{c}_{\widetilde{\varphi}}^{*}\left((F, E)^{c}\right)\right)^{c}$.

The converse of proof is obvious. 
Example 6. Let $X=\left\{x^{1}, x^{2}, x^{3}\right\}, E=\left\{e_{1}, e_{2}\right\}$ and $\widetilde{\varphi}: S S(X)_{E} \rightarrow P\left(S S(X)_{E}\right)$ be a weak soft neighborhood system defined as follows.

$$
\begin{aligned}
& \widetilde{\varphi}\left(x_{e_{1}}^{1}\right)=\left[\left\{\left(e_{1},\left\{x^{1}, x^{3}\right\}\right),\left(e_{2},\left\{x^{1}, x^{2}\right\}\right)\right\}\right], \\
& \widetilde{\varphi}\left(x_{e_{2}}^{1}\right)=\left[\left\{\left(e_{1},\left\{x^{3}\right\}\right),\left(e_{2},\left\{x^{1}\right\}\right)\right\}\right], \\
& \widetilde{\varphi}\left(x_{e_{1}}^{2}\right)=\left[\left\{\left(e_{1},\left\{x^{2}\right\}\right),\left(e_{2},\left\{x^{2}, x^{3}\right\}\right)\right\}\right], \\
& \widetilde{\varphi}\left(x_{e_{2}}^{2}\right)=\left[\left\{\left(e_{1},\left\{x^{1}\right\}\right),\left(e_{2},\left\{x^{2}\right\}\right)\right\}\right], \\
& \widetilde{\varphi}\left(x_{e_{1}}^{3}\right)=\left[\left\{\left(e_{1},\left\{x^{2}, x^{3}\right\}\right),\left(e_{2},\left\{x^{1}, x^{2}, x^{3}\right\}\right)\right\}\right], \\
& \widetilde{\varphi}\left(x_{e_{2}}^{3}\right)=\left[\left\{\left(e_{1}, \emptyset\right),\left(e_{2},\left\{x^{3}\right\}\right)\right\}\right] .
\end{aligned}
$$

Suppose that $(F, E)=\left\{\left(e_{1},\left\{x^{1}, x^{3}\right\}\right),\left(e_{2},\left\{x^{1}, x^{2}\right\}\right)\right\} \widetilde{\subseteq}(X, E)$. Then we have the following results.

(1) Since $\widetilde{J}_{\widetilde{\varphi}}^{*}((F, E))=\left\{\left(e_{1},\left\{x^{1}\right\}\right),\left(e_{2}, \emptyset\right)\right\} \quad$ and $\widetilde{\imath}_{\widetilde{\varphi}}((F, E))=\left\{\left(e_{1},\left\{x^{1}\right\}\right),\left(e_{2},\left\{x^{1}, x^{2}\right\}\right)\right\}$, then $\widetilde{J}_{\widetilde{\varphi}}^{*}((F, E)) \neq \widetilde{\boldsymbol{l}}_{\widetilde{\varphi}}((F, E))$.

(2) Let $(G, E)=(X, E)$. Then $(F, E) \widetilde{\subseteq}(G, E)$ and $\widetilde{J_{\widetilde{\varphi}}^{*}}((G, E))=\Phi$. Thus, $\widetilde{J}_{\widetilde{\varphi}}^{*}((F, E)) \widetilde{\subsetneq} \widetilde{J}_{\widetilde{\varphi}}^{*}((G, E))$.

(3) Let $(G, E)=(X, E)$. Since $\widetilde{J}_{\widetilde{\varphi}}^{*}((F, E) \widetilde{\cap}(G, E))=\left\{\left(e_{1},\left\{x^{1}\right\}\right),\left(e_{2}, \emptyset\right)\right\}$ and $\widetilde{J}_{\widetilde{\varphi}}^{*}((F, E)) \widetilde{\cap} \widetilde{J_{\tilde{\varphi}}^{*}}((G, E))=\Phi$, then $\widetilde{J_{\widetilde{\varphi}}^{*}}((F, E) \widetilde{\cap}(G, E)) \widetilde{\mp} \widetilde{J_{\widetilde{\varphi}}^{*}}((F, E)) \widetilde{\cap} \widetilde{J}_{\widetilde{\varphi}}^{*}((G, E))$.

Theorem 12. Let $(X, \widetilde{\varphi}, E)$ be a WSNS on $(X, E)$ and $(F, E),(G, E) \widetilde{\subseteq}(X, E)$ be two soft sets. Then the following statements are satisfied.

(1) $(F, E) \widetilde{\simeq} \widetilde{c}_{\widetilde{\varphi}}^{*}((F, E))$,

(2) $\widetilde{c}_{\widetilde{\varphi}}^{*}((F, E) \widetilde{\cup}(G, E)) \widetilde{\subseteq} \widetilde{c}_{\widetilde{\varphi}}^{*}((F, E)) \widetilde{\cup} \widetilde{c}_{\widetilde{\varphi}}^{*}((G, E))$,

(3) $\widetilde{c}_{\widetilde{\varphi}}^{*}((F, E))=\left(\widetilde{J}_{\widetilde{\varphi}}^{*}\left((F, E)^{c}\right)\right)^{c}$,

(4) $\widetilde{\kappa}_{\widetilde{\varphi}}((F, E)) \widetilde{\simeq} \widetilde{c}_{\widetilde{\varphi}}^{*}((F, E))$.

Proof. (1), (3) and (4) are obvious.

(2) Let $x_{e} \widetilde{\in} \widetilde{c}_{\widetilde{\varphi}}^{*}((F, E)) \widetilde{\cup} \widetilde{c}_{\widetilde{\varphi}}^{*}((G, E))$. Then $(F, E)^{c}$ and $(G, E)^{c} \in \widetilde{\varphi}\left(x_{e}\right)$. From the property of weak soft neighborhood, $(F, E)^{c} \widetilde{\cap}(G, E)^{c} \in \widetilde{\varphi}\left(x_{e}\right)$. Therefore, $x_{e} \widetilde{\notin} \widetilde{c}_{\widetilde{\varphi}}^{*}((F, E) \widetilde{\cup}(G, E))$.

Theorem 13. Let $(X, \widetilde{\varphi}, E)$ be a WSNS on $(X, E)$ and $(F, E) \widetilde{\subseteq}(X, E)$ be a soft set. Then the following hold:

(1) If $\widetilde{J}_{\widetilde{\varphi}}^{*}((F, E))=(F, E)$, then $(F, E)$ is soft $w_{\widetilde{\varphi}}^{s}-$ open,

(2) If $\widetilde{c}_{\widetilde{\varphi}}^{*}((F, E))=(F, E)$, then $(F, E)$ is soft $w_{\widetilde{\varphi}}^{s}-$ closed.

Proof. (1) Let $\widetilde{J}_{\widetilde{\varphi}}^{*}((F, E))=(F, E)$ for $(F, E) \widetilde{\subseteq}(X, E)$. Since $(F, E)=\widetilde{J}_{\widetilde{\varphi}}^{*}((F, E)) \widetilde{\widetilde{c}} \widetilde{\boldsymbol{l}}_{\widetilde{\varphi}}((F, E))$ and $\widetilde{\boldsymbol{l}}_{\widetilde{\varphi}}((F, E)) \widetilde{\subseteq}(F, E)$, then $\widetilde{\imath}_{\widetilde{\varphi}}((F, E))=(F, E)$. By Theorem 3.8., $(F, E)$ is soft $w_{\widetilde{\varphi}}^{s}-$ open.

(2) Since $\widetilde{c}_{\widetilde{\varphi}}^{*}((F, E))=\left(\widetilde{J}_{\widetilde{\varphi}}^{*}\left((F, E)^{c}\right)\right)^{c}$, then it is obvious.

Definition 34. Let $(X, \widetilde{\varphi}, E),\left(Y, \widetilde{\varphi}^{\prime}, E^{\prime}\right)$ be two WSNS's and $(f, g):(X, \widetilde{\varphi}, E) \rightarrow\left(Y, \widetilde{\varphi}^{\prime}, E^{\prime}\right)$ be a soft mapping. Then $(f, g)$ is called soft $w^{s^{*}}$ - continuous if for $x_{e} \tilde{\in} S S(X)_{E}$ and $(G, E) \in \widetilde{\varphi}^{\prime}\left(f(x)_{g(e)}\right),(f, g)^{-1}((G, E))$ is in $\widetilde{\varphi}\left(x_{e}\right)$.

Every soft $w^{s^{*}}$ - continuous is a soft $w^{s}\left(\widetilde{\varphi}, \widetilde{\varphi}^{\prime}\right)$-continuous, but the following example shows that every soft $w^{s}\left(\widetilde{\varphi}, \widetilde{\varphi}^{\prime}\right)$-continuous function may not be a soft $w^{s^{*}}-$ continuous. 
Example 7. Let $X=\left\{x^{1}, x^{2}\right\}, Y=\left\{y^{1}, y^{2}\right\}$ and $E=\left\{e_{1}, e_{2}\right\}$. Consider two weak soft neighborhood systems $\widetilde{\varphi}$ : $S S(X)_{E} \rightarrow P\left(S S(X)_{E}\right)$ and $\widetilde{\varphi}^{\prime}: S S(Y)_{E} \rightarrow P\left(S S(Y)_{E}\right)$ defined respectively as follows:

$$
\begin{aligned}
& \widetilde{\varphi}\left(x_{e_{1}}^{1}\right)=\left[\left\{\left(e_{1},\left\{x^{1}\right\}\right),\left(e_{2},\left\{x^{1}, x^{2}\right\}\right)\right\}\right], \\
& \widetilde{\varphi}\left(x_{e_{2}}^{1}\right)=\left[\left\{\left(e_{1},\left\{x^{2}\right\}\right),\left(e_{2},\left\{x^{1}\right\}\right)\right\}\right], \\
& \widetilde{\varphi}\left(x_{e_{1}}^{2}\right)=\left[\left\{\left(e_{1},\left\{x^{2}\right\}\right),\left(e_{2},\left\{x^{1}, x^{2}\right\}\right)\right\}\right], \\
& \widetilde{\varphi}\left(x_{e_{2}}^{2}\right)=\left[\left\{\left(e_{1},\left\{x^{1}\right\}\right),\left(e_{2},\left\{x^{2}\right\}\right)\right\}\right],
\end{aligned}
$$

and

$$
\begin{aligned}
& \widetilde{\varphi}^{\prime}\left(y_{e_{1}}^{1}\right)=\left[\left\{\left(e_{1},\left\{y^{1}, y^{2}\right\}\right),\left(e_{2},\left\{y^{1}, y^{2}\right\}\right)\right\}\right], \\
& \widetilde{\varphi}^{\prime}\left(y_{e_{2}}^{1}\right)=\left[\left\{\left(e_{1},\left\{y^{1}, y^{2}\right\}\right),\left(e_{2},\left\{y^{1}\right\}\right)\right\}\right], \\
& \widetilde{\varphi}^{\prime}\left(y_{e_{1}}^{2}\right)=\left[\left\{\left(e_{1},\left\{y^{2}\right\}\right),\left(e_{2},\left\{y^{1}, y^{2}\right\}\right)\right\}\right], \\
& \widetilde{\varphi}^{\prime}\left(y_{e_{2}}^{2}\right)=\left[\left\{\left(e_{1},\left\{y^{1}\right\}\right),\left(e_{2},\left\{y^{2}\right\}\right)\right\}\right] .
\end{aligned}
$$

Let us $(f, g):(X, \widetilde{\varphi}, E) \rightarrow\left(Y, \widetilde{\varphi}^{\prime}, E^{\prime}\right)$ be defined by $f\left(x^{i}\right)_{g\left(e_{i}\right)}=y_{e_{i}}^{i}$ for $i=1,2$ and $x_{e_{i}}^{i} \widetilde{E} S S(X)_{E}$. Then $(f, g)$ is a soft $w^{s}\left(\widetilde{\varphi}, \widetilde{\varphi}^{\prime}\right)$ - continuous but not $w^{s^{*}}-$ continuous.

Corollary 1. From the above results, we have the following implications.

$w^{s^{*}}-$ continuous $\Longrightarrow w^{s}\left(\widetilde{\varphi}, \widetilde{\varphi}^{\prime}\right)-$ continuous $\Longrightarrow w^{s}-$ continuous

Theorem 14. Let $(f, g):(X, \widetilde{\varphi}, E) \rightarrow\left(Y, \widetilde{\varphi}^{\prime}, E^{\prime}\right)$ be a soft mapping on two WSNS's $(X, \widetilde{\varphi}, E)$ and $\left(Y, \widetilde{\varphi}^{\prime}, E^{\prime}\right)$. Then the following conditions are equivalent:

(1) $(f, g)$ is soft $w^{s^{*}}-$ continuous.

(2) For $(G, E) \widetilde{\subseteq}\left(Y, E^{\prime}\right),(f, g)^{-1}\left(\widetilde{J}_{\widetilde{\varphi}^{\prime}}^{*}((G, E)) \widetilde{\subseteq} \widetilde{J}_{\widetilde{\varphi}}^{*}\left((f, g)^{-1}((G, E))\right)\right.$,

(3) For $(G, E) \widetilde{\widetilde{\subseteq}}\left(Y, E^{\prime}\right), \widetilde{c}_{\widetilde{\varphi}}^{*}\left((f, g)^{-1}((G, E))\right) \widetilde{\subseteq}(f, g)^{-1}\left(\widetilde{c}_{\widetilde{\varphi}^{\prime}}^{*}((G, E))\right.$.

Proof. (1) $\Rightarrow(2)$ Let $(f, g)$ be soft $w^{s^{*}}-$ continuous and $x_{e} \widetilde{\in}(f, g)^{-1}\left(\widetilde{J}_{\widetilde{\varphi}^{\prime}}^{*}((G, E))\right.$. Then $(G, E) \in \widetilde{\varphi}^{\prime}\left(f(x)_{g(e)}\right)$. From the definition of soft $w^{s^{*}}$ continuous, $(f, g)^{-1}((G, E)) \in \widetilde{\varphi}\left(x_{e}\right)$. Hence, $x_{e} \widetilde{\in} \widetilde{J}_{\widetilde{\varphi}}^{*}\left((f, g)^{-1}((G, E))\right)$.

(2) $\Rightarrow(3)$ It is obtained from the Definition 33 .

(3) $\Rightarrow(1)$ It is obvious by the Theorem 33. and the Theorem 12 .

\section{Conclusions}

In this paper, the concepts of weak soft neighborhood space, soft $w^{s}\left(\widetilde{\varphi}, \widetilde{\varphi}^{\prime}\right)$-continuous, soft $w^{s}$-continuous and soft $w^{s^{*}}$ - continuous on weak soft neighborhood spaces are defined. We introduce their basic theorems and some interesting results. By using same idea, we can define soft $w^{s}\left(\widetilde{\varphi}, \widetilde{\varphi}^{\prime}\right)$ - open functions, soft $w^{s}-$ open functions and soft $w^{s^{*}}-$ open functions on weak soft neighborhood spaces.

\section{References}

[1] Aktas H. and Çağman N., Soft sets and soft group, Information Science 177 (2007) 2726-2735. 
[2] Bayramov S., Gunduz (Aras) C. and Demirci N., A new approach to inverse and direct systems of soft topological spaces, Maejo International Journal of Science and Technology, 10(01) (2016) 51-65.

[3] Bayramov S. and Gunduz (Aras) C., Soft locally compact spaces and soft paracompact spaces, Journal of Mathematics and System Science, 3 (2013) 122-130.

[4] Császár Á., Generalized topology, generalized continuity, Acta. Math. Hungar. 96 (2002) 351-357.

[5] Çağman N., Karatas S. and Enginoğlu S., Soft topology, Comput. Math. Appl. (2011) 351-358.

[6] Gunduz A. C., Sonmez A. and Çakallı H., On soft Mappings, (to appear).

[7] Maji P. K., Bismas R. and Roy A. R., Soft Set Theory, Comput. Math. Appl. 45 (2003) 555-562.

[8] Min W. K., On weak neighborhood systems and spaces, Acta. Math. Hungar. 121 (3) (2008) 283-292.

[9] Molodtsov D., Soft Set Theory-First Results, Comput. Math. Appl.37 (1999) 19-31.

[10] Ozturk T. Y. and Bayramov S., Soft mapping spaces, The Scientific World Journal, Article ID 307292, (2014) 8p.

[11] Shabir M. and Naz M., On soft topological spaces, Comput. Math. Appl. 61 (2011) 1786-1799.

[12] Shabir M. and Bashir A., Some properties of soft topological spaces, Comput. Math. Appl. 62 (2011) 4058-4067.

[13] Sahin R. and Küçük A., Soft Filters and Their Convergence Properties, Annals of Fuzzy Mathematics and Informatics 6(3) (2013) 529-543.

[14] Sahin R., Soft compactification of soft topological spaces: Soft star topological spaces, Annals of Fuzzy Mathematics and Informatics 10(2) (2015) 447-464.

[15] Thomas J. and John J. S., On soft generalized topological spaces, Journ. of New Results in Sci. 4 (2014) 01-15. 